\title{
Correction to: General practitioners' and out-of-hours doctors' role as gatekeeper in emergency admissions to somatic hospitals in Norway: registry-based observational study
}

\author{
Jesper Blinkenberg ${ }^{1,2^{*}}$, Sahar Pahlavanyali ${ }^{1,2}, \varnothing$ ystein Hetlevik ${ }^{2}$, Hogne Sandvik $^{1}$ and Steinar Hunskaar ${ }^{1,2}$
}

\author{
Correction to: BMC Health Serv Res 19, 568 (2019) \\ https://doi.org/10.1186/s12913-019-4419-0
}

Following publication of the original article [1], the authors would like to correct several numbers in the following paragraphs. In addition, Fig. 1, Fig. 2, Fig. 3, Table 1, Table 2, Table 3, and Table 4 need to be corrected as well.

The numbers need to be corrected for two reasons, both technical and originating from the preparation of the research data files. First, the authors discovered that the material missed data for $8 \%$ of primary care contacts due to a data transfer error between the university and the main public data registry. Second, technical personnel in our data centre had made an error in the algorithm when linking datasets, leading to less prehospital contacts linked to emergency hospital admissions. As a result, most numbers in the Results, Tables and Figures were affected. Most of the changes were, however, of insignificant magnitude, and the errors did not affect the main conclusions of the article.

The updated paragraphs are given below, and include the whole results and discussion section, as well as all tables and figures.

The original article can be found online at https://doi.org/10.1186/s12913 019-4419-0.

* Correspondence: jesper.blinkenberg@norceresearch.no

${ }^{1}$ National Centre for Emergency Primary Health Care, NORCE Norwegian

Research Centre, Kalfarveien 31, 5018 Bergen, Norway

${ }^{2}$ Department of Global Public Health and Primary Care, University of Bergen,

Kalfarveien 31, 5018 Bergen, Norway

\section{Results in the Abstract:}

Results: In 2014 there were 497,845 emergency admissions to somatic hospitals in Norway after excluding birth related conditions. Referrals by $\mathrm{OOH}$ doctors were most frequent (36\%), 35\% were direct admissions, $28 \%$ were referred by GPs, whereas only $2 \%$ were referred from outpatient clinics or private specialists with public contract. Direct admissions were more common in central areas (45\%), here GPs' referrals constituted only $18 \%$. The prehospital paths varied with the hospital discharge diagnosis. For anaemias, $52-56 \%$ were referred by GPs, for acute appendicitis and mental/alcohol related disorders $57 \%$ and $56 \%$ were referred by $\mathrm{OOH}$ doctors, respectively. For malignant neoplasms 56\% and cardiac arrest 57\% were direct admissions

\section{Results in main text:}

\section{Results}

There were 551,753 emergency hospital admissions to somatic hospitals in Norway in 2014, according to our case definition. One in ten admissions were birth related, hence not supposed to have visited a primary healthcare doctor before admission (Fig. 1). After excluding the birth-related admissions from the material, the distribution of the remaining 497,845 somatic emergency hospital admissions by referring agents is shown in Fig. 1. Referrals by $\mathrm{OOH}$ doctors were most frequent (36\%), 35\% were direct admissions, $28 \%$ were referred by GPs, whereas only $2 \%$ were referred from outpatient's clinics or PSPCs. 


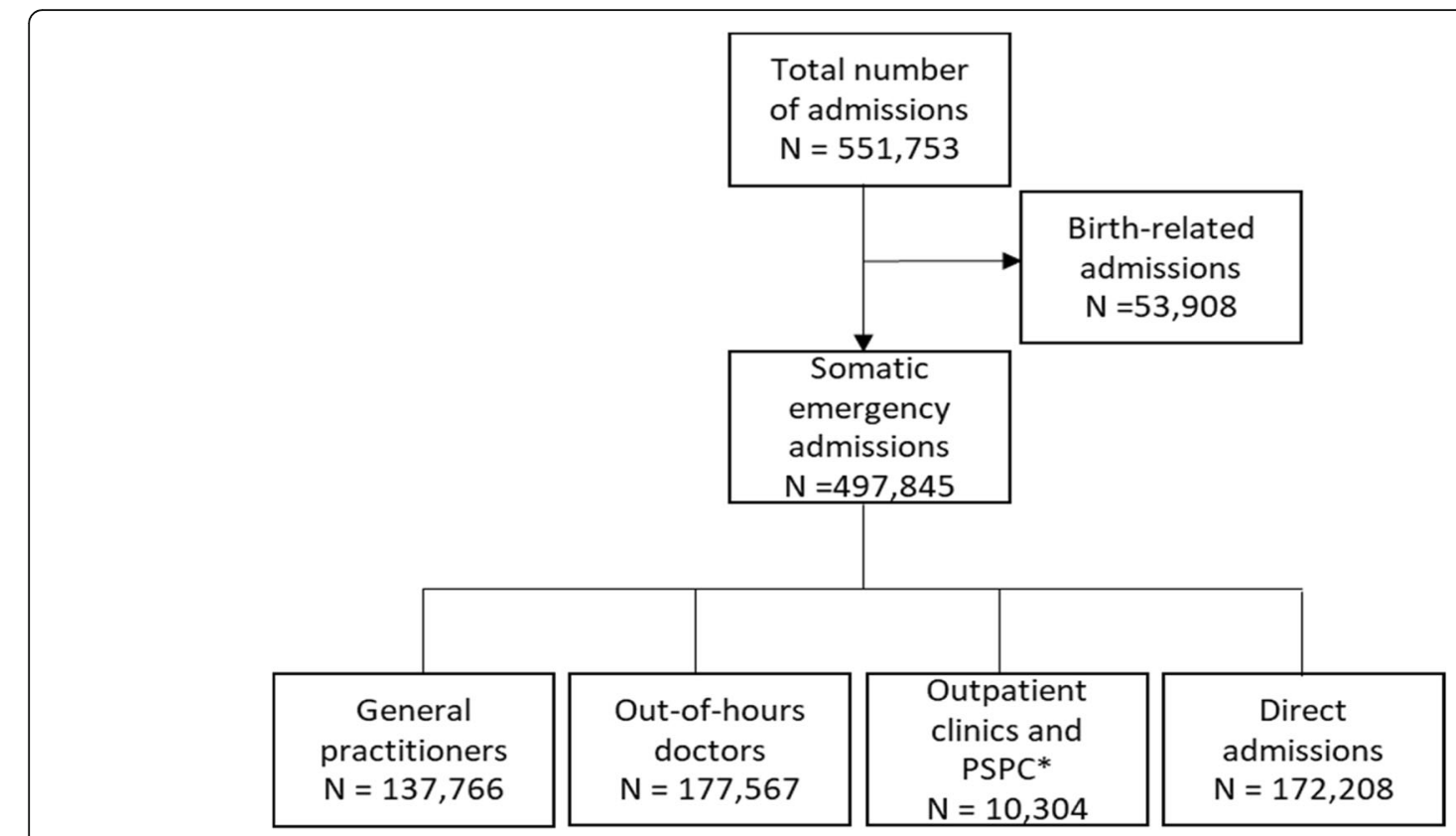

Fig. 1 Prehospital pathways for all the emergency admissions to somatic hospitals in Norway in 2014 *Private specialist with public contract

\section{Day and time of admission}

Large differences in prehospital paths were found for weekdays vs. weekends, and by day and night hours (Fig. 2). On weekdays, most patients were admitted during the daytime, $53 \%$ from 8 am to $4 \mathrm{pm}$.
GP contacts were the main prehospital path in this period, with a little dip representing lunch hour. No patients were admitted from GPs during weekends. Patients referred from the $\mathrm{OOH}$ services were the largest group during evenings and nights on weekdays,

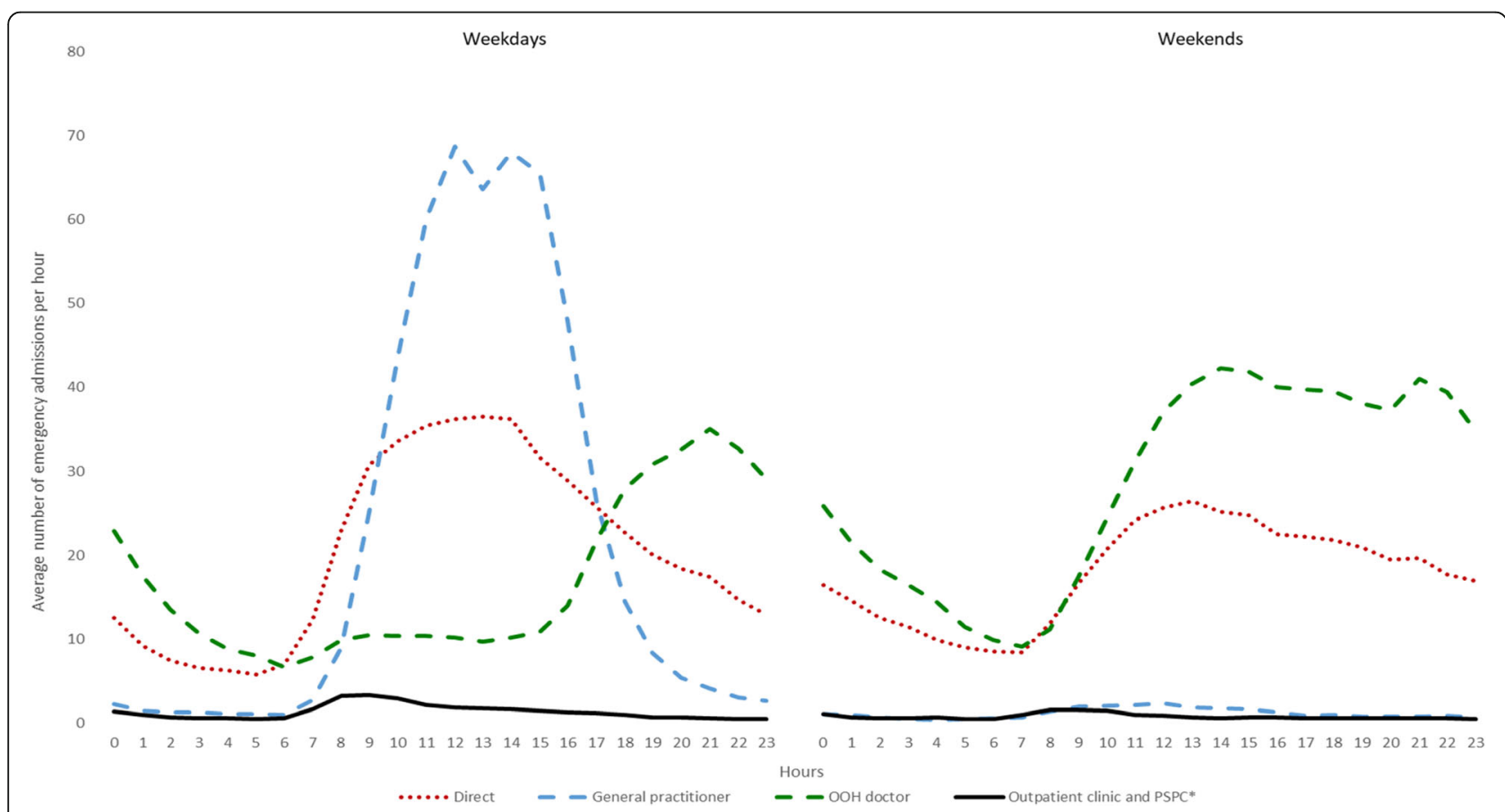

Fig. 2 All emergency admissions to somatic hospitals in 2014 in Norway, sorted by prehospital pathways and time of the day during weekdays and weekends 
and all weekends. Direct admissions were high during morning hours and midday, both weekdays and weekends.

\section{Centrality patterns}

Table 1 and Table 2 show emergency admissions by centrality group, referring agent, and per 1000 inhabitants. The mean number of emergency admissions per 1000 inhabitants per year was 97, highest in the least central group (115), and lowest in the most central group (87). For direct admissions, we found an increasing proportion by increasing centrality, so in the most central (urban) areas almost half of the admissions to somatic hospitals in 2014 were direct admissions. For the two least central areas, with $12 \%$ of the population and $14 \%$ of the admissions, only $28 \%$ of the admissions were direct.

There was an increasing proportion of referrals from GPs by decreasing centrality, as referrals from GPs constituted only $18 \%$ in the most central group and $34 \%$ of the admissions in the two least central groups of municipalities. The proportion of patients referred from $\mathrm{OOH}$ doctors was relatively stable by centrality group, varying from 32 to $37 \%$ in the various centrality groups. Outpatient clinics and PSPCs referred few patients, and had low shares in all centrality groups, but reached $4 \%$ in the most central group. Hospitals in the most central regions had up to $57 \%$ direct admissions, whereas the most rural had only $22 \%$ (data not shown).

\section{Diagnoses}

Among all the emergency admissions, injuries were the most frequent discharge diagnosis group, followed by diseases in the circulatory system, symptoms and findings not elsewhere classified, and diseases in the respiratory system (Fig. 3).

Table 3 shows the 20 most common diagnoses by the four prehospital paths, these diagnoses constituted 35\% of all admissions. Pneumonia (J15, J18) was the most common diagnosis, followed by pain in throat and chest (R07), abdominal and pelvic pain (R10), atrial arrhythmias (I48), and acute myocardial infarction (I21). Several

Table 1 Frequency of all emergency admissions to somatic hospitals in Norway 2014 by patient residence centrality

\begin{tabular}{|c|c|c|c|c|}
\hline \multirow[t]{2}{*}{ Centrality } & \multicolumn{2}{|c|}{ All admissions } & \multicolumn{2}{|c|}{ Population } \\
\hline & $\mathrm{N}$ & $\%$ & $\mathrm{~N}$ & Admissions per 1000 \\
\hline 1 (most central) & 88,086 & 18 & $1,011,602$ & 87 \\
\hline 2 & 122,043 & 25 & $1,199,290$ & 102 \\
\hline 3 & 124,055 & 25 & $1,357,164$ & 91 \\
\hline 4 & 94,456 & 19 & 906,580 & 104 \\
\hline 5 & 48,982 & 10 & 459,368 & 107 \\
\hline 6 (least central) & 20,107 & 4 & 175,052 & 115 \\
\hline Sum & $497,729^{a}$ & 100 & $5,109,056$ & 97 \\
\hline
\end{tabular}

${ }^{\mathrm{a}} 116$ cases missing the centrality variable kinds of injuries were also in the top 20, together with major chronic diseases such as chronic obstructive pulmonary disease (COPD) and heart failure.

Prehospital paths differed considerably between different discharge diagnoses (Table 4). The GPs $(28 \%$ of all emergency admissions) had a much higher share of, e.g. anaemias and other conditions of the blood, sciatica, heart failure, and various local subacute diseases like haemorrhoids, diverticulitis, and deep venous thrombosis. $\mathrm{OOH}$ doctors (36\% of all admissions) had a high share of referrals for various acute conditions, like appendicitis, foreign body in alimentary tract, mental and alcohol related disorders, abdominal pain and other acute gastro-intestinal conditions, asthma, and nephrolithiasis. The direct prehospital path (35\% of all admissions) was most common for the diagnosis of agranulocytosis, hydrocephalus and cardiac arrest, but all with relatively small absolute numbers. The top seven diagnoses with direct admissions had a percentage above 50, revealing a list of conditions being extensively removed from undergoing a gatekeeper process. Admissions for malignant neoplasms was by far the largest group $(C)(56 \%, N=24,190)$, followed by fractures and other orthopedic conditions, epilepsy, and chronic diseases of the lungs, kidneys and heart. Major and common emergencies, such as stroke (42\%), acute myocardial infarction (42\%) and pneumonia (29\%) did not reach the top 20 list of direct admissions but had high absolute numbers.

\section{Discussion in main text \\ Main results}

We found that $28 \%$ of emergency-admitted patients to somatic hospitals in Norway in 2014 were referred by a GP and $36 \%$ by an $\mathrm{OOH}$ doctor. The second largest group of patients were admitted without a registered contact prior to admission (direct admission, 35\%). While referrals from GPs were most frequent during office hours, $\mathrm{OOH}$ doctors referred patients mainly during evenings, nights and weekends. Direct admissions had the same diurnal pattern as the total emergency admissions, more admissions in daytime and less during the night. Fewer patients living in the most central region were referred by GPs than in less central regions (18\% versus $27-34 \%$ ). More patients were directly admitted $(45 \%)$ in the most central areas.

When analysing the prehospital paths for different discharge diagnoses, we found considerable variation. It is likely that the explanation for this lies in the nature of the clinical presentation and urgency of the medical conditions, in addition to health service factors. Similar to the findings of Vest-Hansen et al. in Denmark, this study showed that pneumonia was the most common admitted emergency medical condition (25). 
Table 2 Variation in prehospital paths by patient residence centrality for all emergency admissions to somatic hospitals in Norway $2014\left(N=497,729^{\mathrm{a}}\right)$

\begin{tabular}{|c|c|c|c|c|c|c|c|c|}
\hline \multirow[b]{2}{*}{ Centrality } & \multicolumn{2}{|c|}{ General practitioner } & \multicolumn{2}{|c|}{ Out-of-hours doctor } & \multicolumn{2}{|c|}{ Outpatient clinic or PSPC ${ }^{\mathbf{b}}$} & \multicolumn{2}{|c|}{ Direct admission } \\
\hline & $\mathrm{N}$ & $\%$ & $\mathrm{~N}$ & $\%$ & $\mathrm{~N}$ & $\%$ & $\mathrm{~N}$ & $\%$ \\
\hline 1 (most central) & 15,820 & 18 & 28,596 & 32 & 3828 & 4 & 39,842 & 45 \\
\hline 2 & 32,363 & 27 & 45,181 & 37 & 2098 & 2 & 42,401 & 35 \\
\hline 3 & 36,372 & 29 & 43,537 & 35 & 2045 & 2 & 42,101 & 34 \\
\hline 4 & 29,651 & 31 & 34,676 & 37 & 1537 & 2 & 28,592 & 30 \\
\hline 5 & 16,708 & 34 & 18,104 & 37 & 606 & 1 & 13,564 & 28 \\
\hline 6 (least central) & 6845 & 34 & 7424 & 37 & 189 & 1 & 5649 & 28 \\
\hline
\end{tabular}

${ }^{a} 116$ cases missing the centrality variable

${ }^{\text {bPrivate specialist with public contract }}$

\section{Strengths and limitations}

Our study includes all residents of Norway, and all their GP- and $\mathrm{OOH}$ contacts, and all emergency admissions to somatic hospitals in 2014. Hence, there is no selection bias. The registries used are based on data delivered with the purpose of managing funding of primary- and specialist healthcare and are therefore probably complete. This means that the material is fully representative for Norway.
There is no information of referring services in the NPR, and we therefore had to make an algorithm for this purpose. The algorithm linked $65 \%$ of all emergency admissions to a referring service. Some of the prehospital contacts categorized as referring contacts might be random contacts with no connection to the admission. Nevertheless, we found a clear accumulation of contacts within the $24 \mathrm{~h}$ before admission, reducing the likeliness for high incidence of random linkage. Some prehospital

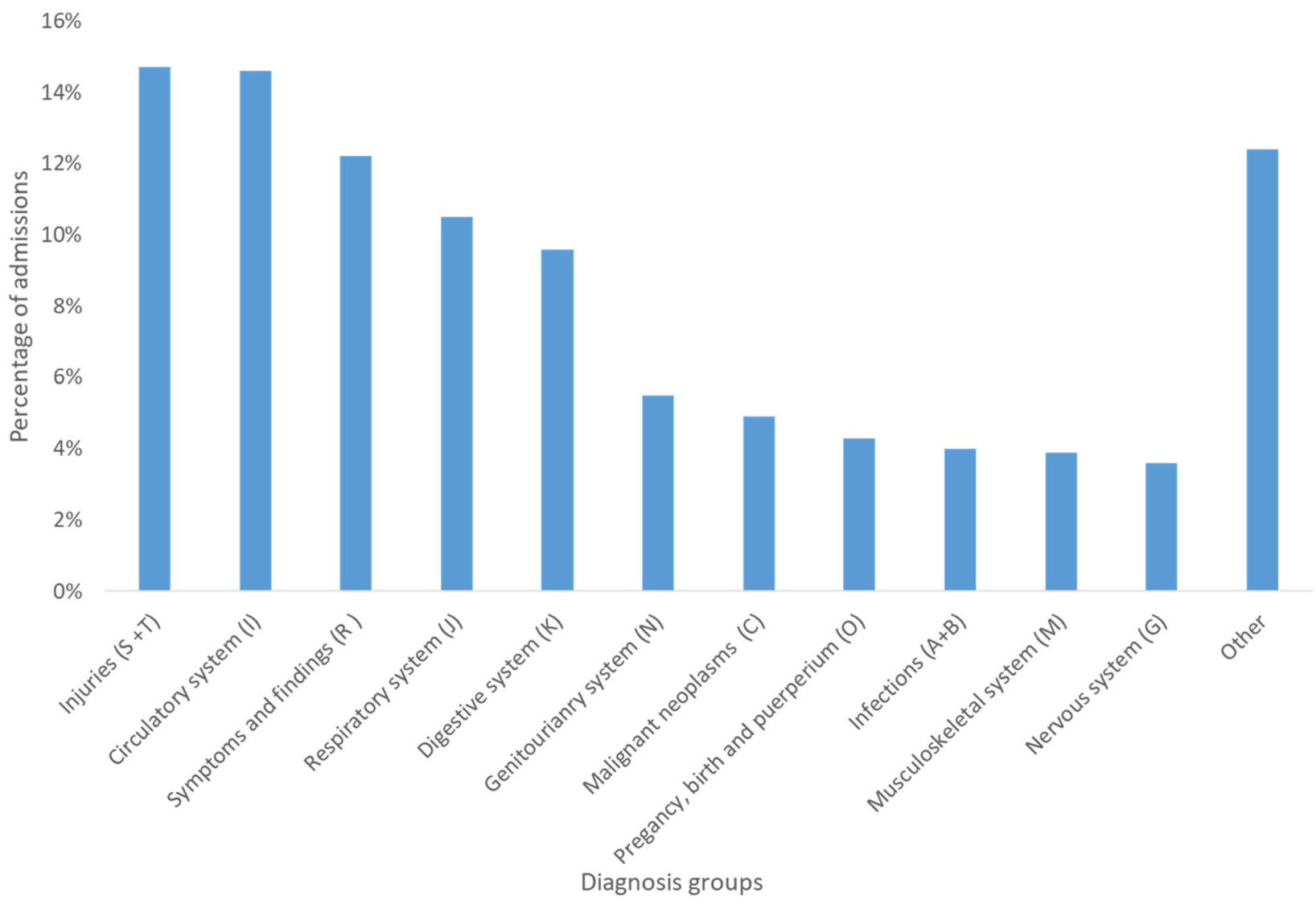

Fig. 3 Distribution of admissions by diagnosis groups for the discharge diagnosis (ICD-10) after emergency admissions to somatic hospitals (except normal birth and related conditions) in Norway 2014 ( $N=497,845)$ 
Table 3 Distribution of prehospital pathways for all admissions (except birth related conditions), and by discharge diagnosis (ICD-10 codes) for the 20 most common diagnosis after somatic hospital stays in Norway 2014

\begin{tabular}{|c|c|c|c|c|c|c|c|c|c|c|}
\hline & \multicolumn{2}{|c|}{$\begin{array}{l}\text { General } \\
\text { practitioner }\end{array}$} & \multicolumn{2}{|c|}{$\begin{array}{l}\text { Out-of-hours } \\
\text { doctor }\end{array}$} & \multicolumn{2}{|c|}{$\begin{array}{l}\text { Outpatient clinic or } \\
\text { PSPC }^{\mathbf{a}}\end{array}$} & \multicolumn{2}{|c|}{$\begin{array}{l}\text { Direct } \\
\text { admission }\end{array}$} & \multicolumn{2}{|l|}{ Sum } \\
\hline & $\mathrm{N}$ & $\%$ & $\mathrm{~N}$ & $\%$ & $\mathrm{~N}$ & $\%$ & $\mathrm{~N}$ & $\%$ & $\mathrm{~N}$ & $\%$ \\
\hline All admissions & 137,766 & 28 & 177,567 & 36 & 10,304 & 2 & 172,208 & 35 & 497,845 & 100 \\
\hline \multicolumn{11}{|l|}{ Diagnosis (ICD-10) } \\
\hline Pneumonia (J15 + J18) & 6499 & 32 & 7918 & 39 & 109 & 1 & 5962 & 29 & 20,488 & 100 \\
\hline Pain in throat and chest (R07) & 4710 & 29 & 7613 & 47 & 74 & 0 & 3923 & 24 & 16,320 & 100 \\
\hline Abdominal and pelvic pain (R10) & 4930 & 32 & 7874 & 51 & 81 & 1 & 2633 & 17 & 15,518 & 100 \\
\hline Atrial fibrillation and flutter (148) & 4423 & 37 & 3885 & 33 & 170 & 1 & 3395 & 29 & 11,873 & 100 \\
\hline Acute myocardial infarction (I21) & 2699 & 24 & 3814 & 34 & 92 & 1 & 4705 & 42 & 11,310 & 100 \\
\hline Fracture of femur (S72) & 1634 & 16 & 3417 & 34 & 192 & 2 & 4715 & 47 & 9958 & 100 \\
\hline Chronic obstructive pumonary disease (J44) & 2743 & 30 & 3461 & 38 & 45 & 0 & 2754 & 31 & 9003 & 100 \\
\hline Intracranial injury (S06) & 1178 & 14 & 3734 & 45 & 316 & 4 & 3021 & 37 & 8249 & 100 \\
\hline Other dissorders of urinary system (N39) & 2233 & 30 & 3158 & 42 & 49 & 1 & 2058 & 27 & 7498 & 100 \\
\hline Cerebral infarction (163) & 1973 & 27 & 2313 & 31 & 45 & 1 & 3078 & 42 & 7409 & 100 \\
\hline Heart failure (150) & 2935 & 40 & 2191 & 30 & 72 & 1 & 2194 & 30 & 7392 & 100 \\
\hline Angina pectoris (I20) & 2107 & 31 & 2253 & 33 & 113 & 2 & 2277 & 34 & 6750 & 100 \\
\hline Complications of procedures (T81) & 1257 & 22 & 1581 & 27 & 174 & 3 & 2808 & 48 & 5820 & 100 \\
\hline Alcohol related disorders (F10) & 641 & 11 & 3262 & 56 & 24 & 0 & 1852 & 32 & 5779 & 100 \\
\hline Acute appendicitis (K35) & 1827 & 32 & 3233 & 57 & 9 & 0 & 573 & 10 & 5642 & 100 \\
\hline Syncope and collapse (R55) & 1305 & 25 & 2240 & 42 & 45 & 1 & 1704 & 32 & 5294 & 100 \\
\hline Choleolithiasis (K80) & 1549 & 31 & 2488 & 50 & 22 & 0 & 943 & 19 & 5002 & 100 \\
\hline Medical observation (Z03) & 1567 & 32 & 1735 & 35 & 52 & 1 & 1560 & 32 & 4914 & 100 \\
\hline Fracture of forearm (S52) & 698 & 15 & 1992 & 42 & 317 & 7 & 1770 & 37 & 4777 & 100 \\
\hline Fracture of lower leg, including ancle (S82) & 630 & 13 & 1858 & 40 & 211 & 5 & 1983 & 42 & 4682 & 100 \\
\hline Sum & 47,538 & & 70,020 & & 2212 & & 53,908 & & 173,678 & 35 (of all) \\
\hline
\end{tabular}

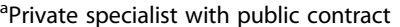

contacts with GP or $\mathrm{OOH}$ services may not provide sufficient help, leading patients to contact EMCC, which might result in a direct admission by ambulance services. However, only for the most urgent cases would this comply with the national admission routines.

We used the discharge diagnosis to describe the medical condition for each admission. This does not give accurate information about the clinical presentation at the time of admission, which is the basis for deciding the prehospital path. Using the referral diagnosis from the gatekeeping GP and $\mathrm{OOH}$ doctor could put extra information on this, but the $35 \%$ direct admission would not have such a referral diagnosis. Reasons for encountering GPs or $\mathrm{OOH}$ services are not generally available in Norway, and it is thus not possible to link e.g. abdominal pain, fever, etc. to the referral situation.

\section{Gatekeeping}

Generally, a gatekeeping system gives power to primary care doctors (GPs and $\mathrm{OOH}$ doctors) to decide whether a patient needs specialty care, hospital care, or a diagnostic test, and patients not have access to specialist or hospital care without a prior examination and a referral (26). Gatekeeping is associated with lower utilization of health services and has been suggested to reduce hospitalizations (15). In a healthcare system facing capacity problems, this is a preferred development. Recently there has been debate on the value of gatekeeping related to GPs' workload and patient choice (14). Although Norway has a gatekeeperbased healthcare system, we found that only $65 \%$ of the emergency-admitted patients came through the primary healthcare gatekeeping system. This is in line with the findings of Grondal et al. from a smaller study at a medical department in Norway, where GPs and $\mathrm{OOH}$ doctors referred 26 and 31\%, respectively (17). A reasonable level of gatekeeping for emergency admissions is not possible to determine. However, the variation by centrality could indicate that primary 
Table 4 Emergency admissions by discharge ICD-10 diagnosis where contact with a) GP or b) out-of-hour (OOH) doctor, or c) direct admission is the dominating prehospital pathway

a) GP contact before admission $(N=137,766)$

\section{Diagnosis}

Iron deficiency anaemia (D50)

Other anaemias (D64)

Haemorrhoids (K64)

Diverticular disease (K57)

Abscess of anal and rectal regions (K61)

Intervertebral disc disorders (M51)

Localized swelling, head (R22)

Phlebitis and thrombophlebitis (180)

Gout (M10)

Mononucleosis (B27)

Other spondylopathies (M48)

Venous embolism and thrombosis (182)

Excessive vomiting in pregnancy (O21)

Malaise and fatigue (R53)

Ulcerative colitis (K51)

Heart failure (150)

Atherosclerosis (170)

Disturbances of skin sensation (R20)

Facial nerve disorders (G51)

Osteomyelitis (M86)

\section{Admissions with the discharge diagnose}

N

1980

1274

655

3234

1214

2180

523

1428

659

517

735

548

1205

516

969

7392

1097

745

516

526

\section{GP contact before admission}

Admissions with the discharge diagnose

N

690

1717

732

5642

5779

757

1130

1129

1995

1433

15,518

5002

1419

1065

3225

3356

2017

2100
$\%$

56

52

48

48

47

47

46

46

44

43

43

43

42

41

40

40

39

39

39

39

$\mathrm{OOH}$ contact before admission

$\%$ 60

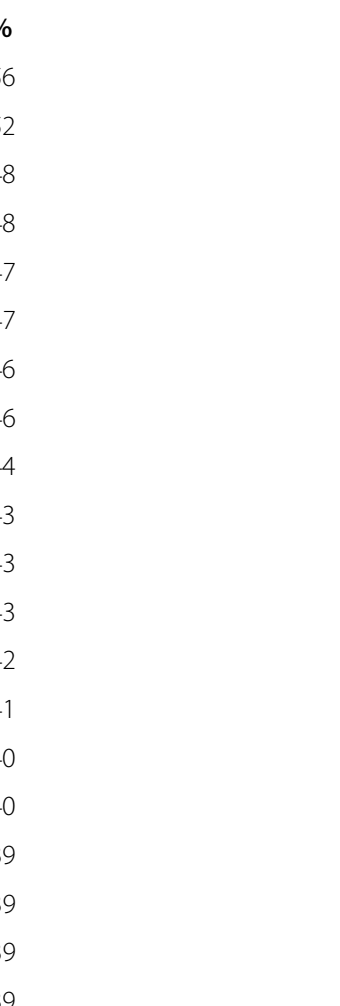
58 58 57 56 54 53 53 52 51 51 50 50 49 49 48 48 48

Asthma (J45) 
Table 4 Emergency admissions by discharge ICD-10 diagnosis where contact with a) GP or b) out-of-hour (OOH) doctor, or c) direct admission is the dominating prehospital pathway (Continued)

\begin{tabular}{|c|c|c|}
\hline Dorsalgia (M54) & 3648 & 47 \\
\hline Calculus of kidney (N20) & 3324 & 47 \\
\hline \multicolumn{3}{|c|}{$\begin{array}{l}\text { c) Direct admissions except the ICD-10 diagnosis groups pregnancy, childbirth and the puerperium (0XX), and factors influencing health } \\
\text { status and contact with health services }(Z X X)(N=172,208)\end{array}$} \\
\hline & Admissions with the discharge diagnose & Direct admission \\
\hline Diagnosis & $\mathbf{N}$ & $\%$ \\
\hline Agranulocytosis (D70) & 749 & 66 \\
\hline Hydrocephalus (G91) & 587 & 64 \\
\hline Cardiac arrest (146) & 539 & 57 \\
\hline Malignant neoplasms (C) & 24,190 & 56 \\
\hline Orthopaedic complications (T84) & 2001 & 54 \\
\hline Superficial injury of thorax (S20) & 522 & 53 \\
\hline Intracerebral haemorrhage (161) & 1421 & 51 \\
\hline Mental/sedatives dissorders (F13) & 658 & 49 \\
\hline Open wound of head (S01) & 849 & 49 \\
\hline Multiple sclerosis (G35) & 969 & 49 \\
\hline Complications of procedures ICA (T81) & 5820 & 48 \\
\hline Epilepsy (G40) & 3874 & 48 \\
\hline Fracture of femur (S72) & 9958 & 47 \\
\hline Chronic ischaemic heart disease (125) & 2954 & 47 \\
\hline Aortic aneurysm and dissection (171) & 982 & 46 \\
\hline Fracture of skull and facial bones (S02) & 1132 & 45 \\
\hline Pleural effusion, not elsewhere classified (J90) & 915 & 45 \\
\hline Nonrheumatic aortic valve disorders (I35) & 1280 & 44 \\
\hline Convulsions, not elsewhere classified (R56) & 1838 & 44 \\
\hline Pneumonitis due to food and vomit (J69) & 836 & 44 \\
\hline
\end{tabular}

care doctor gatekeeping can be obtained for two thirds of emergency admissions. This could reduce the workload and expenses in hospital care (14).

The diagnoses where the GP played a major role as gatekeeper in our material were anaemias, of which 52$56 \%$ of the patients were referred by GP, infections (39$47 \%)$ and worsening of chronic disease (39-44\%). These diagnoses seem to be less urgent, and might be identified at a regular control consultation, or an extra emergency contact at the GP office. This resembles the picture from Denmark where anaemia, diabetes, atrial fibrillation and heart failure show a reduction in admission rate from office-hours when GPs work, to evening, night and weekend (25). Skarshaug et al. found a similar pattern in another Norwegian study, showing that $74 \%$ of the patients admitted with heart failure had a GP contact within the previous month (27).

The $\mathrm{OOH}$ doctor more often was referring patients with conditions where medical investigation and treatment is more urgent, like abdominal pain (47-57\%) and mental illness/substance abuse and intoxication (54-56\%).

\section{Direct admissions}

The direct admissions are the second most frequent prehospital path in our material, and may represent admissions from nursing homes, admissions initiated by hospital doctors following up the patients in specialist healthcare, or directly admitted by ambulance services. As expected, direct admissions are more frequent for highly urgent conditions such as cardiac arrest (57\%) and intracerebral haemorrhage (51\%) suggesting direct admissions by ambulance service. Our study also shows that 43 and $49 \%$ of these cases, respectively, do have a GP or $\mathrm{OOH}$ contact before admission. According to national guidelines, cerebral infarction should be managed by direct prehospital path (28). However, 27\% were referred by GPs and $31 \%$ by $\mathrm{OOH}$ doctors. A study from The Netherlands found that as many as $49 \%$ of patients with acute stroke had a GP contact before admission (29). Probably, some of these patients contact their GP or other primary care providers instead of EMCC in emergencies. The clinical presentation of such urgent 
conditions is not always the classic acute pattern, similar to stroke and acute coronary syndrome (29, 30).

On the other hand, we know that the $\mathrm{OOH}$ doctors and GPs are highly involved in acute cases. In 2014, 65\% of the Norwegian $\mathrm{OOH}$ services reported that the doctors participate in emergency callouts always or often, when alarmed (22). One earlier study showed that GPs or $\mathrm{OOH}$ doctors participated in $42 \%$ of alerted emergency cases $(31,32)$. In 2015, the new emergency medicine regulation in Norway stated that the $\mathrm{OOH}$ doctors are obliged to be contacted in the emergency communication system and to participate in emergency callouts, when needed (21).

Some medical conditions are followed up in specialist care at hospitals. It is likely that worsening or complications may be discovered at specialist care consultations, or by the patient's direct contact to the hospital. This might contribute to the high proportion of direct admissions for malignant neoplasms (56\%) and orthopaedic complications (54\%). Grondal et al. found that $18 \%$ of all admissions to a medical department were from outpatient clinics and open return agreements (17). It is likely that admissions from outpatient clinics at the hospital are often converted for administrative reasons directly from an outpatient contact to an emergency admission without registering the outpatient clinic contact. Also, some of the patients with a discharge diagnosis of malignant disease might have been admitted because of acute symptoms, and then diagnosed with cancer during the hospital stay. Again, these patients would, according to national procedures, usually have been guided by the EMCC or $\mathrm{OOH}$ services to a primary care doctor to get a medical examination and referral.

Hip fracture (S72) had a high proportion of direct admissions (47\%), illustrating a condition where GP or $\mathrm{OOH}$ consultation often is not necessary in order to reveal the need for hospital care. This supports the finding of Skarshaug et al. where $50 \%$ of patients urgently admitted to hospital with hip fracture had no GP or $\mathrm{OOH}$ contact the month prior to emergency admission (27).

Referrals from nursing home doctors are not specified in our material but included in the direct admissions. We found the same proportion of direct admissions for elderly patients as for the total population, 80-89 years 33 , and $34 \%$ for patients 90 years and older. This indicates that admissions from nursing home doctors do not significantly affect the proportion of direct admissions.

\section{Time of the day}

The gatekeeping function was delivered by the GPs and $\mathrm{OOHs}$ doctor according to activity in the services, $\mathrm{GP}$ in the opening hours, and $\mathrm{OOH}$ doctors the rest of the week. The gatekeeper activity is higher than direct admissions throughout the day, with a period in the morning, both on weekdays and weekends, where the direct admissions are as frequent as GP and $\mathrm{OOH}$ referrals. This might be because some emergencies are discovered in the morning when the patient and the relatives wake up, or by that the $\mathrm{OOH}$ and GP services have less capacity in the transition time between nightshift and daytime work.

\section{Centrality}

GPs and $\mathrm{OOH}$ doctors participate less in the emergency callouts in the most central regions in Norway $(31,32)$. This may explain the low gatekeeper activity of GPs in the central area, but we did not find the same effect for $\mathrm{OOH}$ doctors. Thus, hyper-acute cases with callouts represent relatively few admissions, and therefore the effect of this is relatively sparse. The GPs' low share of referrals to hospitals may rather be due to GPs in most central regions being less accessible for urgent consultations than their more rural colleagues, but this is not possible to investigate in the present study. Unlike Bankart et al. we found higher rates of emergency admissions in rural areas (7).

\section{Interpretations}

Based on our findings, Norwegian GPs and $\mathrm{OOH}$ doctors are gatekeepers in fewer emergency admissions to somatic hospitals than expected, when taking into account the rather strict gatekeeping system that is principally in place. The direct prehospital path representing admissions from ambulance services, referrals from nursing home doctors, and admissions initiated by hospital doctors, represent a large part of the emergency admissions. This should be taken into account when planning health care services, including strategies in order to reduce hospital overload. On the other hand, there are many clinical conditions where both GPs' and $\mathrm{OOH}$ doctors' gatekeeping role are considerable.

Published online: 16 September 2020

\section{Reference}

1. Blinkenberg, et al. General practitioners' and out-of-hours doctors' role as gatekeeper in emergency admissions to somatic hospitals in Norway: registry-based observational study. BMC Health Serv Res. 2019;19:568 https://doi.org/10.1186/s12913-019-4419-0. 